TRANSACTIONS OF THE

AMERICAN MATHEMATICAL SOCIETY

Volume 356, Number 3, Pages 1185-1207

S 0002-9947(03)03432-9

Article electronically published on September 22, 2003

\title{
COMPLETE HYPERELLIPTIC INTEGRALS OF THE FIRST KIND AND THEIR NON-OSCILLATION
}

\author{
LUBOMIR GAVRILOV AND ILIYA D. ILIEV
}

\begin{abstract}
Let $P(x)$ be a real polynomial of degree $2 g+1, H=y^{2}+P(x)$ and $\delta(h)$ be an oval contained in the level set $\{H=h\}$. We study complete Abelian integrals of the form

$$
I(h)=\int_{\delta(h)} \frac{\left(\alpha_{0}+\alpha_{1} x+\ldots+\alpha_{g-1} x^{g-1}\right) d x}{y}, h \in \Sigma,
$$

where $\alpha_{i}$ are real and $\Sigma \subset \mathbb{R}$ is a maximal open interval on which a continuous family of ovals $\{\delta(h)\}$ exists. We show that the $g$-dimensional real vector space of these integrals is not Chebyshev in general: for any $g>1$, there are hyperelliptic Hamiltonians $H$ and continuous families of ovals $\delta(h) \subset\{H=h\}$, $h \in \Sigma$, such that the Abelian integral $I(h)$ can have at least $\left[\frac{3}{2} g\right]-1$ zeros in $\Sigma$. Our main result is Theorem 1 in which we show that when $g=2$, exceptional families of ovals $\{\delta(h)\}$ exist, such that the corresponding vector space is still Chebyshev.
\end{abstract}

\section{INTRODUCTION}

Take real polynomials $H, f, g \in \mathbb{R}[x, y]$ and let $\delta(h) \subset\left\{(x, y) \in \mathbb{R}^{2}: H(x, y)=\right.$ $h\}, h \in \Sigma$, be a continuous family of ovals. For sufficiently small $\varepsilon$ and generic $H, f, g$, the limit cycles of the perturbed plane Hamiltonian system

$$
d H+\varepsilon(f d x+g d y)=0
$$

which tend to certain ovals from the continuous family when $\varepsilon \rightarrow 0$, are in one-toone correspondence with the zeros of the complete Abelian integral

$$
I(h)=\int_{\delta(h)} f(x, y) d x+g(x, y) d y, \quad h \in \Sigma .
$$

For this reason the problem of finding the zeros of $I(h)$ in terms of the degrees of $H, f, g$ was called by Arnold [5, p. 313] the "weakened 16th Hilbert problem" (compare to Hilbert [19]; see also Arnold [6], 7], 8]). Note that the level sets $\{H=h\}$ will contain in general several continuous families of ovals which need be considered separately.

It follows from the Varchenko-Khovanskii theorem that the number of the zeros of $I(h)$ is bounded by a constant $N(n, d)<\infty$, uniformly in all $H, f, g$, such that $\operatorname{deg} f \leq d$, deg $g \leq d$, and $\operatorname{deg} H \leq n$.

In the so-called "hyperelliptic case" $\left(H=y^{2}+P(x)\right)$ it was proved by Novikov and Yakovenko[21] that there exists an algorithm producing a function $C(n, d)$ such

Received by the editors December 18, 2002.

2000 Mathematics Subject Classification. Primary 34C08; Secondary 14D05, 14K20, 34C07.

(C)2003 American Mathematical Society 
that $\infty>C(n, d)>N(n, d)$, which is given by a tower function (an iterated exponent) of height at least five. Their proof is based on the analytic properties of a suitable Picard-Fuchs system satisfied by Abelian integrals (including the magnitude of the coefficients of the system and some restrictions on its monodromy group).

The progress in solving the weakened 16th Hilbert problem (finding the exact number of the zeros of $I(h)$ ) has thus far only concerned the "elliptic case" (the complex algebraic curve $\{H=h\}$ is of genus at most one). It was proved for instance that in several cases, the vector space $\mathcal{A}_{H, d}$ of Abelian integrals of degree $d$ polynomials along the ovals of $H$, obeys the so-called Chebyshev property (the number of the zeros of each integral is smaller than the dimension of the vector space $\left.\mathcal{A}_{H, d}\right)$; see [22, 15], 14], [11. In this relation Arnold asked in [8, the 7th problem] whether the $g$-dimensional vector space of Abelian integrals

$$
I(h)=\int_{\delta(h)} \frac{\left(\alpha_{0}+\alpha_{1} x+\ldots+\alpha_{g-1} x^{g-1}\right) d x}{y}, h \in \Sigma,
$$

where $\delta(h) \subset\left\{(x, y) \in \mathbb{R}^{2}: y^{2}+P(x)=h\right\}, g=\left[\frac{1}{2}(\operatorname{deg} P-1)\right]>1$, is Chebyshev.

In an attempt to solve this problem Givental [17. obtained a non-oscillation theorem for Lagrangian planes of the Picard-Fuchs system satisfied by the Abelian integrals $\int_{\delta(h)} x^{i} d x / y$. He used the fact that every Picard-Fuchs system has a Hamiltonian (or Poisson) structure. He failed, however, to produce bounds for the zeros of the Abelian integrals.

In the present paper we try to explore the algebro-geometrical properties of the Abelian integrals, without using the Picard-Fuchs system. Our main result is Theorem 1 in which we show that when $g=2$ and $\operatorname{deg} P=5$, exceptional families of ovals $\{\delta(h)\}, h \in \Sigma$, exist such that every Abelian integral of the form

$$
I(h)=\int_{\delta(h)} \frac{\left(\alpha_{0}+\alpha_{1} x\right) d x}{y}, \quad \alpha_{0}^{2}+\alpha_{1}^{2} \neq 0,
$$

has at most one simple zero on the interval $\Sigma$. At a first sight this seems to be an easy observation (equivalent to the claim that $\left(\int_{\delta(h)} x d x / y\right) /\left(\int_{\delta(h)} d x / y\right)$ is monotonous on $\Sigma$ ). This is, however, the first result of such a kind for non-elliptic curves $\{H=h\}$. The proof uses the Riemann bilinear relations on differentials of the first kind together with the fact that a Jacobian variety with its polarization cannot be a direct product of principally polarized Abelian varieties. Our arguments can be adapted to other situations (for instance when the degree of $P(x)$ is 6 ), but we shall not do this here.

We give also a negative answer (Proposition 2) to the initial question posed by Arnold: it turns out that there exist Abelian integrals of the form (1) with exactly $\left[\frac{3}{2} g\right]-1$ zeros in a neighborhood of the origin. At the end of the paper we discuss in brief some more general problems we hope are appropriate for a future research and formulate two hypotheses concerning Chebyshev accuracy in the case $\operatorname{deg} P=5$.

\section{THE CYCLICITY AT A CENTER}

In this section we prove that in general, the integral (1) does not belong to a Chebyshev space. Consider first the particular case when $n=5$. Let $H=y^{2}+P(x)$ where $P(x)=x^{2}\left(1+a_{1} x+a_{2} x^{2}+a_{3} x^{3}\right), a_{1}, a_{2}, a_{3} \in \mathbb{R}, a_{3} \neq 0$, and denote by 
$\delta(t) \subset\left\{y^{2}+P(x)=t\right\}$ a continuous family of cycles vanishing at the origin as $t$ tends to zero.

Proposition 1. The Abelian integral

$$
I(t)=\int_{\delta(t)} \frac{\left(\alpha_{0}+\alpha_{1} x\right) d x}{y}=\alpha_{0} I_{0}(t)+\alpha_{1} I_{1}(t) \not \equiv 0
$$

can have a zero at the origin of multiplicity at most two. Moreover, for fixed $\alpha_{1}, a_{2}, a_{3}, \alpha_{1} a_{3} \neq 0$, there exist a sufficiently small $\varepsilon>0$ and $\alpha_{0}, a_{1}$ in a small neighborhood of zero, such that $I(t)$ has exactly two simple zeros in the interval $(0, \varepsilon)$.

The above shows that the real vector space generated by the functions $I_{0}(t)$, $I_{1}(t)$ is not Chebyshev. Recall that a real vector space $V$ of functions defined on some interval $\Sigma$ is said to be Chebyshev, provided that each $f \in V$ has at most $\operatorname{dim} V-1$ zeros (counted with multiplicity) on $\Sigma$, and Chebyshev with accuracy $m$, if each $f \in V$ has at most $\operatorname{dim} V+m-1$ zeros there.

Proof of Proposition 1. For a small $x$, denote $X=x\left(1+a_{1} x+a_{2} x^{2}+a_{3} x^{3}\right)^{1 / 2}$. Then an easy calculation yields the inverse transformation

$x=\varphi(X) \sim X-\frac{1}{2} a_{1} X^{2}+\left(\frac{5}{8} a_{1}^{2}-\frac{1}{2} a_{2}\right) X^{3}-\left(a_{1}^{3}-\frac{3}{2} a_{1} a_{2}+\frac{1}{2} a_{3}\right) X^{4}+\ldots \quad$ as $\quad X \rightarrow 0$.

Therefore, for small positive $t$,

$$
\begin{aligned}
& I(t)=\int_{y^{2}+X^{2}=t} \frac{\left[\alpha_{0}+\alpha_{1} \varphi(X)\right] \varphi^{\prime}(X)}{y} d X \\
& =\int_{y^{2}+X^{2}=t} \frac{\alpha_{0}\left[1+O\left(X^{2}\right)\right]-\alpha_{1}\left[\frac{3}{2} a_{1} X^{2}+\frac{5}{2}\left(\frac{21}{8} a_{1}^{3}-\frac{7}{2} a_{1} a_{2}+a_{3}\right) X^{4}+O\left(X^{6}\right)\right]}{y} d X \\
& =2 \pi \alpha_{0}[1+O(t)]-2 \pi \alpha_{1}\left[\frac{3}{4} a_{1} t+\frac{15}{16}\left(\frac{21}{8} a_{1}^{3}-\frac{7}{2} a_{1} a_{2}+a_{3}\right) t^{2}+O\left(t^{3}\right)\right] .
\end{aligned}
$$

When $\alpha_{0}=a_{1}=0$, the function $I(t)$ has a double zero at the origin since $\alpha_{1} a_{3} \neq 0$. Taking $\alpha_{1}=1,\left|\alpha_{0}\right| \ll\left|a_{1}\right| \ll\left|a_{3}\right|$ and $\alpha_{0} a_{1}>0>a_{1} a_{3}$, one finishes the proof of the proposition.

Clearly, the above approach could be applied to hyperelliptic Hamiltonians of any degree that have a center.

Denote by $\mathcal{H}_{n}$ the set of Hamiltonians $H=y^{2}+x^{2}+a_{1} x^{3}+\ldots+a_{n-2} x^{n}$, where $a_{k} \in \mathbb{R}, a_{n-2} \neq 0$. Denote by $g=\left[\frac{1}{2}(n-1)\right] \geq 2$ the genus of the hyperelliptic curve. Given $H \in \mathcal{H}_{n}$, let $\delta(t), t \in\left(0, T_{H}\right)$, be an oval from the continuous family of ovals surrounding the center at the origin. Consider the vector space $\mathcal{A}_{H}$ formed by the Abelian integrals

$$
I(t)=\int_{\delta(t)} \frac{G(x) d x}{y}, \quad t \in\left(0, T_{H}\right), \quad G \in \mathbb{R}[x], \quad \operatorname{deg} G<g .
$$

Let us point out that $\operatorname{dim} \mathcal{H}_{H}=g$ in general, but $\operatorname{dim} \mathcal{H}_{H}=\left[\frac{1}{2}(g+1)\right]$ provided that $H$ contains no odd degree monomials. In the proposition below, we give a partial answer to the question raised by V. Arnold'd in [8].

Proposition 2. There exist $H \in \mathcal{H}_{n}$ and a polynomial $G$ such that the corresponding integral (2) has exactly $\left[\frac{3}{2} g\right]-1$ small positive simple zeroes. Thus, such a space $\mathcal{A}_{H}$ could be Chebyshev with accuracy at least $\left[\frac{1}{2} g\right]$ in $\left(0, T_{H}\right)$. 
Given an integer $k \geq 0$ and $H \in \mathcal{H}_{n}$, denote

$$
I_{k}(t)=\int_{\delta(t)} \frac{x^{k} d x}{y}, \quad 0<t<T_{H}
$$

The proof of the above proposition is based on the following lemma.

Lemma 1. The following asymptotic expansions hold for small positive t:

(i) $I_{2 k}(t)=t^{k}\left[c_{k}+O(t)\right]$, where $c_{k}=2 \pi \frac{(2 k-1) ! !}{(2 k) ! !}$,

(ii) $I_{2 k+1}(t)=-\frac{1}{2} \sum_{j=1}^{g} a_{2 j-1}(2 k+2 j+1) t^{k+j}\left[c_{k+j}+O(t)\right]$,

therefore the coefficients in the last expansion belong to the ideal generated by the odd-numbered parameters $\left\{a_{1}, a_{3}, \ldots, a_{2 g-1}\right\}$.

Proof. Take as above $X=x\left(1+a_{1} x+\ldots+a_{n-2} x^{n-2}\right)^{1 / 2}$. Then the inverse transformation $x=\varphi\left(X, a_{1}, a_{2}, \ldots, a_{n-2}\right)$ for small $X$ reads $x=X+A_{1} X^{2}+A_{2} X^{3}+$ $A_{3} X^{4}+\ldots$, where the coefficients $A_{j}=A_{j}\left(a_{1}, a_{2}, \ldots, a_{n-2}\right)$ are polynomials. It is easy to see that if one attaches a weight $k$ to the coefficient $a_{k}$, then $A_{j}$ is a polynomial of weight $j$. Indeed, directly from the definition of $\varphi$ it follows that

$$
\varphi\left(\omega X, a_{1}, a_{2}, \ldots, a_{n-2}\right)=\omega \varphi\left(X, \omega a_{1}, \omega^{2} a_{2}, \ldots, \omega^{n-2} a_{n-2}\right) .
$$

Applying this identity to

$$
\varphi\left(X, a_{1}, \ldots, a_{n-2}\right)=X\left(1+\sum_{k=1}^{\infty} A_{k}\left(a_{1}, \ldots, a_{n-2}\right) X^{k}\right),
$$

we get $A_{k}\left(\omega a_{1}, \ldots, \omega^{n-2} a_{n-2}\right)=\omega^{k} A_{k}\left(a_{1}, \ldots, a_{n-2}\right)$. To calculate the coefficient at $a_{k}$ in $A_{k}$, we take $a_{1}=\ldots=a_{k-1}=0$, and easily obtain that it equals $-\frac{1}{2}$. Therefore, one deduces that $A_{k}=-\frac{1}{2} a_{k}+A_{k}^{*}$ where $A_{k}^{*}$ belongs to the polynomial ideal generated by $a_{1}, \ldots, a_{k-1}$.

In case (i), one immediately obtains that for small positive $t$,

$$
I_{2 k}(t)=\frac{1}{2 k+1} \int_{y^{2}+X^{2}=t} \frac{d \varphi^{2 k+1}(X)}{y}=\int_{y^{2}+X^{2}=t} \frac{\left(X^{2 k}+\ldots\right) d X}{y}=c_{k} t^{k}+O\left(t^{k+1}\right) .
$$

To calculate the integral in case (ii), we write $\varphi^{2 k+2}(X)$ in the form

$$
\begin{gathered}
\varphi^{2 k+2}=X^{2 k+2}\left(1+\sum_{j=1}^{\infty}\left(-\frac{1}{2} a_{j}+A_{j}^{*}\right) X^{j}\right)^{2 k+2} \\
=X^{2 k+2}\left(1+\sum_{j=1}^{\infty}\left[(2 k+2)\left(-\frac{1}{2} a_{j}+A_{j}^{*}\right) X^{j}+O\left(X^{j+1}\right)\right]\right) \\
=X^{2 k+2}\left(1-\sum_{j=1}^{n-2}(k+1) a_{j} X^{j}[1+O(X)]\right) \\
=-(k+1) \sum_{j=1}^{g} a_{2 j-1} X^{2 k+2 j+1}\left[1+O\left(X^{2}\right)\right]+\Phi(X),
\end{gathered}
$$


where $\Phi(X)$ is a series in $X$ containing only even order powers. Therefore,

$$
\begin{gathered}
I_{2 k+1}(t)=\frac{1}{2 k+2} \int_{y^{2}+X^{2}=t} \frac{d \varphi^{2 k+2}(X)}{y} \\
=-\frac{1}{2} \sum_{j=1}^{g} a_{2 j-1}(2 k+2 j+1) t^{k+j}\left[c_{k+j}+O(t)\right] .
\end{gathered}
$$

Proof of Proposition [. Denote $m=\left[\frac{1}{2} g\right]$ and take in (2)

$$
G(x)=\sum_{k=0}^{m-1} \gamma_{2 k} x^{2 k}-x^{2 m-1} .
$$

Then by Lemma 1,

$$
I(t)=\sum_{k=0}^{m-1} \gamma_{2 k} t^{k}\left[c_{k}+O(t)\right]+\frac{1}{2} \sum_{j=1}^{g} a_{2 j-1}(2 m+2 j-1) t^{m+j-1}\left[c_{m+j-1}+O(t)\right] .
$$

Now, freeze the even-numbered coefficients in $H$ and then choose $\gamma_{2 j}$ and $a_{2 j-1}$ so that $\gamma_{2 j} \gamma_{2 j+2}<0, a_{2 j-1} a_{2 j+1}<0, \gamma_{2 m-2} a_{1}<0$ and

$$
\left|\gamma_{0}\right| \ll\left|\gamma_{2}\right| \ll \ldots \ll\left|\gamma_{2 m-2}\right| \ll\left|a_{1}\right| \ll \ldots \ll\left|a_{2 g-1}\right| \ll 1 .
$$

Such a choice guarantees that $I(t)$ will have exactly $m+g-1=\left[\frac{3}{2} g\right]-1$ zeroes in a certain small interval $(0, \varepsilon)$.

This result raises the problem to describe the hyperelliptic Hamiltonians $H$ and the continuous families of ovals $\{\delta(h)\}$ for which the space of integrals (1) obeys the Chebyshev property. In the rest of the paper, we concentrate our efforts on the simplest case when $\operatorname{deg} P=5$. We begin with some preliminaries.

\section{The NORMAL FORM, THE BIFURCATION DIAGRAM AND THE DYNKIN DIAGRAM FOR $n=5$}

We consider the hyperelliptic Hamiltonian $H=\frac{1}{2} y^{2}+P(x)$, where $P \in \mathbb{R}[x]$ and $\operatorname{deg} P=5$. Assume that a certain level set $\{H=h\}$ contains an oval (compact closed smooth curve without critical points). As the Poincaré index of the respective Hamiltonian vector field $X_{H}$ is zero, then $X_{H}$ has at least two different real critical points. Without any loss of generality, we can place all of the four critical points at $(0,0),(\mu, 0),(\lambda, 0)$ and $(1,0)$, where either $0 \leq \mu \leq \lambda \leq 1$ (the real case) or $\bar{\mu}=\lambda \in \mathbb{C} \backslash \mathbb{R}$ (the complex case). Finally, we can assume that the coefficient at $x^{5}$ is positive. Then after rescaling of the $y$ variable, we come to the normal form

$$
H(x, y)=\frac{1}{2} y^{2}-\frac{\lambda \mu}{2} x^{2}+\frac{\lambda+\mu+\lambda \mu}{3} x^{3}-\frac{1+\lambda+\mu}{4} x^{4}+\frac{1}{5} x^{5} \equiv \frac{1}{2} y^{2}+P(x),
$$

and the Hamiltonian flow $X_{H}$ takes the form

$$
\begin{aligned}
& \dot{x}=y, \\
& \dot{y}=-x(x-\mu)(x-\lambda)(x-1) .
\end{aligned}
$$

Until the end of the paper, we will only use this normal form of $H$.

Clearly, the origin is a hyperbolic saddle if $\mu \neq 0$, and $(1,0)$ is a nondegenerate center provided that $\lambda \neq 1$. In addition, when $0<\mu<\lambda<1$ in the real case, $(\mu, 0)$ 
is a center and $(\lambda, 0)$ is a saddle. Denote by $h_{0}, h_{\mu}, h_{\lambda}$ and $h_{1}$ the corresponding critical levels of $H$. One has

$$
\begin{aligned}
& h_{0}=0, \quad h_{1}=-\frac{1}{60}(3-5 \lambda-5 \mu+10 \lambda \mu), \\
& h_{\lambda}=-\frac{\lambda^{3}}{60}\left(3 \lambda^{2}-5 \lambda \mu-5 \lambda+10 \mu\right), \\
& h_{\mu}=-\frac{\mu^{3}}{60}\left(3 \mu^{2}-5 \lambda \mu-5 \mu+10 \lambda\right) .
\end{aligned}
$$

If $0<\mu<\lambda<1$, we have $h_{0}>h_{\mu}, h_{\lambda}>h_{\mu}, h_{\lambda}>h_{1}$. The bifurcation diagram $D$ in the real case consists of the boundary of the triangle $T=\{0 \leq \mu \leq \lambda \leq 1\}$ and the curve $\gamma: h_{\lambda}=h_{0}$ having an equation

$$
\mu=\frac{3 \lambda^{2}-5 \lambda}{5(\lambda-2)}
$$

see Figure 1

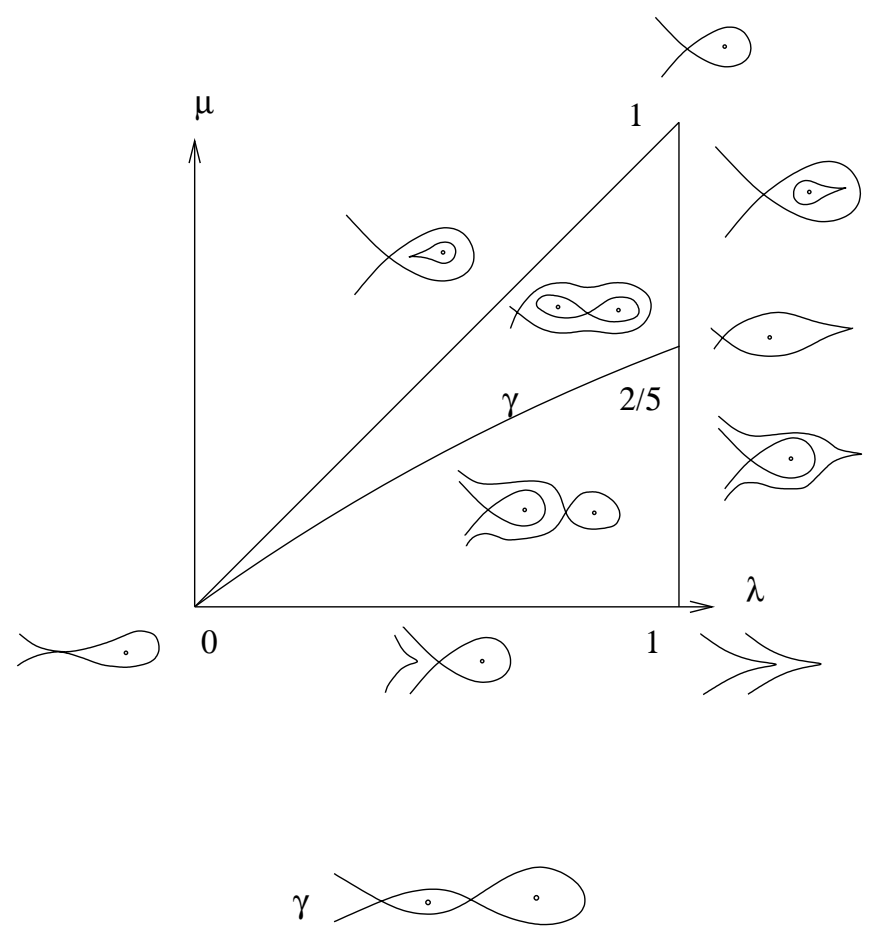

Figure 1. Bifurcation diagram in the $(\lambda, \mu)$-plane in the real case.

There are two open components in $T \backslash D$ giving the two generic cases. Namely, for parameters $\lambda, \mu$ above the curve $\gamma, X_{H}$ has three period annuli, and below this curve $X_{H}$ has just two period annuli. There are also five codimension-one and four codimension-two degenerate (non-generic) cases corresponding to the parameters on the bifurcation diagram $D$. In the complex case, there is a unique period annulus around $(1,0)$ which terminates at the saddle-loop through $(0,0)$ and the phase portraits for all $\lambda \in \mathbb{C} \backslash \mathbb{R}$ are topologically equivalent. We will denote the period annuli (continuous families of ovals) around $(1,0),(\mu, 0)$ and the eight loop by $\mathcal{O}_{1}$, $\mathcal{O}_{\mu}$ and $\mathcal{O}_{e}$, respectively. 


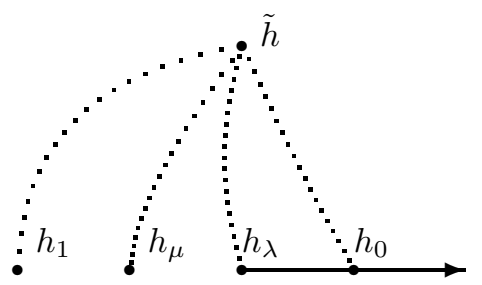

(i)

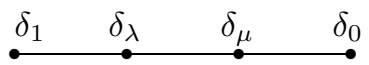

(ii)

Figure 2. (i) The paths $l_{0}, l_{1}, l_{\lambda}, l_{\mu}$ in the real case $0<\mu<\lambda<1$ when $h_{1}<h_{\mu}<h_{\lambda}<h_{0}$; (ii) Dynkin diagram in the real case $0<\mu<\lambda<1$.

Below we describe the Dynkin diagram of the real polynomial $H(x, y)$ when the critical levels are distinct. Recall that it is a graph defined in the same way as the Dynkin diagram (or D-diagram) of a germ of an analytical function with an isolated singularity; see [3], [10]. The vertices of the diagram are in one-toone correspondence with the vanishing cycles of the polynomial. Two vertices are connected by an edge if the intersection number of the cycles is not zero. To an edge one also associates a label equal to the intersection number, but it will be omitted here because it is of no importance for us. The vanishing cycles "vanish" along suitable paths, and hence the Dynkin diagram also depends on the family of paths. The precise definition in our situation is as follows.

First consider the real case $0<\mu<\lambda<1$. There are five possible distributions of the critical values as follows:

$$
\begin{array}{ll}
h_{1}<h_{\mu}<h_{\lambda}<h_{0}, & h_{1}<h_{\mu}<h_{0}<h_{\lambda}, \quad h_{\mu}<h_{1}<h_{0}<h_{\lambda}, \\
h_{\mu}<h_{0}<h_{1}<h_{\lambda}, & h_{\mu}<h_{1}<h_{\lambda}<h_{0} .
\end{array}
$$

For definiteness, assume that $h_{1}<h_{\mu}<h_{\lambda}<h_{0}$. Denote $\mathcal{D}=\mathbb{C} \backslash\left[h_{\lambda}, \infty\right)$ and let

$$
l_{0}, l_{1}, l_{\lambda}, l_{\mu}:[0,1] \rightarrow\{h \in \mathbb{C}: \operatorname{Im} h \geq 0\}
$$

be continuous paths connecting some fixed regular value $\tilde{h} \in \mathcal{D}$ to $h_{0}, h_{1}, h_{\lambda}, h_{\mu}$ respectively, as shown in Figure 2.

The Dynkin diagram of $H$ with respect to the above paths is a graph with four vertices corresponding to the four families of cycles vanishing along the paths $l_{0}$, $l_{1}, l_{\lambda}$, and $l_{\mu}$, which we denote by $\delta_{0}(h), \delta_{1}(h), \delta_{\mu}(h)$, and $\delta_{\lambda}(h)$, respectively. Two vertices are connected by an edge if the intersection number of the cycles for $h=\tilde{h}$ is not zero (in which case it is \pm 1 and may be supposed equal to one). The computation of the Dynkin diagram of a real polynomial with real critical points only is well known; see for instance [1], [3]. It yields the diagram shown in Figure 2 . We note that the computation depends only on the distribution of the four critical points $0<\mu<\lambda<1$ and not on the distribution of the respective critical values (hence, the Dynkin diagram is the same for all possible arrangements of the critical values with corresponding paths $\left.l_{0}, l_{1}, l_{\lambda}, l_{\mu}\right)$. 


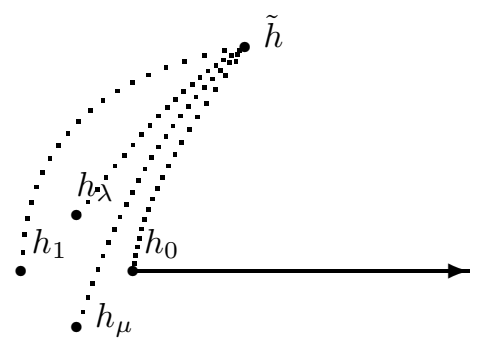

(i)

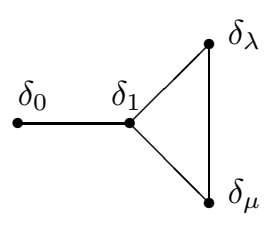

(iii)

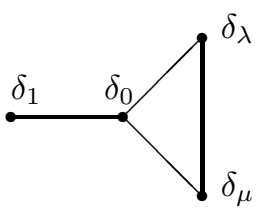

(ii)

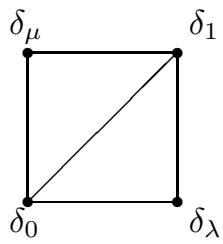

(iv)

Figure 3. (i) The paths $l_{0}, l_{1}, l_{\lambda}, l_{\mu}$ in the case when $\lambda \notin \mathbb{R}$ and $h_{\lambda} \neq h_{\mu}$; (ii) Dynkin diagram in the case when $\lambda \notin \mathbb{R}$ lies inside the left branch of the hyperbola $\Gamma$; (iii) Dynkin diagram in the case when $\lambda \notin \mathbb{R}$ lies inside the right branch of the hyperbola $\Gamma$; (iv) Dynkin diagram in the case when $\lambda \notin \mathbb{R}$ lies between the two branches of the hyperbola $\Gamma$.

Now consider the complex case when $\lambda=\bar{\mu} \notin \mathbb{R}$. The method we use is a deformation of $H$ to a polynomial having real critical points. Denote $\mathcal{D}=\mathbb{C} \backslash\left[h_{0}, \infty\right)$ and let

$$
l_{0}, l_{1}, l_{\lambda}, l_{\mu}:[0,1] \rightarrow \mathcal{D}
$$

be continuous paths connecting some fixed regular value $\tilde{h} \in \mathcal{D}$ to $h_{0}, h_{1}, h_{\lambda}, h_{\mu}$, respectively, as shown in Figure 3. The Dynkin diagram related to these paths is defined as above. Consider in the complex plane the hyperbola $\Gamma$ defined by the equation

$$
\Gamma: h_{\lambda}=h_{\mu} \quad \Leftrightarrow \quad(\operatorname{Im} \lambda)^{2}=5 \operatorname{Re} \lambda(\operatorname{Re} \lambda-1)
$$

and drawn in Figure 5 .

Suppose for definiteness that $\lambda$ lies inside its left branch. First, by continuous deformation of $\lambda=\bar{\mu}$ such that in the course of the deformation $\lambda$ remains in the left branch of (5), we may achieve that $\lambda=\mu<0<1$. This, combined with $h_{\lambda}=h_{\mu}<0$, already implies that

- the intersection number of $\delta_{1}(\tilde{h})$ and $\delta_{0}(\tilde{h})$ is equal to \pm 1 ; 
- the intersection number of $\delta_{\lambda}(\tilde{h}), \delta_{\mu}(\tilde{h})$ with $\delta_{1}(\tilde{h})$ is zero;

- the intersection number of $\delta_{\lambda}(\tilde{h})$ and $\delta_{\mu}(\tilde{h})$ is equal to \pm 1 ;

- the intersection number of either $\delta_{\lambda}(\tilde{h})$ or $\delta_{\mu}(\tilde{h})$ with $\delta_{0}(\tilde{h})$ is equal to \pm 1 .

Finally, we may use the real structure of the complex algebraic curve

$$
\Gamma_{h}=\left\{(x, y) \in \mathbb{C}^{2}: H(x, y)=h\right\}
$$

for $h \in \mathbb{R}$. This structure is defined by an antiholomorphic involution $i$ on the curve which is the complex conjugation. It induces on its hand an involution $i_{*}$ of $H_{1}\left(\Gamma_{h}, \mathbb{Z}\right),\left(i_{*}\right)^{2}=i d$. It is easy to see that for $h<0$ we have

$$
i_{*} \delta_{\lambda}(h)=\delta_{\mu}(h), \quad i_{*} \delta_{0}(h)=-\delta_{0}(h) .
$$

This combined with the identity

$$
\overline{\langle\delta(h), \gamma(h)\rangle}=-\left\langle i_{*} \delta(h), i_{*} \gamma(h)\right\rangle
$$

shows that

$$
\left\langle\delta_{\lambda}(h), \delta_{0}(h)\right\rangle=\left\langle\delta_{\mu}(h), \delta_{0}(h)\right\rangle .
$$

This implies the Dynkin diagram as shown in Figure 3(ii)). The remaining cases shown there are studied in a similar way.

We finish this section by introducing a property of the continuous families of ovals which is dependent on the structure of the related Dynkin diagram. Suppose that the real polynomial $H=y^{2}+P(x)$ has distinct critical values. Let $\delta(h) \subset\{H=h\}$ be a continuous family of ovals defined on a maximal open interval $\Sigma=\left(h_{c}, h_{s}\right)$, where for $h=h_{c}$ the oval degenerates to a point $\delta\left(h_{c}\right)$ which is a center and for $h=h_{s}$ the oval becomes a homoclinic loop of the Hamiltonian system $d H=0$. The family $\{\delta(h)\}$ represents a continuous family of cycles vanishing at the center $\delta\left(h_{c}\right)$. To formulate our main result we shall need the following

Definition 1. It is said that $\{\delta(h)\}$ is an exceptional family of ovals, provided that for every polynomial one-form $\omega$ the Abelian integral

$$
I(h)=\int_{\delta(h)} \omega, \quad h \in \Sigma,
$$

allows an analytic continuation in the sector $S_{\varepsilon}\left(h_{s}\right)=\left\{h \in \mathbb{C}: \operatorname{Arg}\left(h-h_{s}\right) \in\right.$ $(-\varepsilon, 2 \pi+\varepsilon)\}$ for some strictly positive $\varepsilon$. The corresponding family of vanishing cycles represented by $\delta(h)$ is called an exceptional family of vanishing cycles.

The above definition has in fact a geometric nature. Indeed, $I(h)$ has an analytic continuation in the sector $S_{\varepsilon}\left(h_{s}\right)$ if and only if $\delta(h)$ has an appropriate intersection number with each of the other families of vanishing cycles. For instance, if $\lambda, \mu$ are real and $h_{1}<h_{\mu}<h_{\lambda}<h_{0}$, then the family of cycles $\delta_{1}(h)$ is exceptional, because it has a zero intersection number with $\delta_{0}(h)$. On the contrary, the family $\delta_{\mu}(h)$ is not exceptional, because it has a non-zero intersection number with $\delta_{0}(h)$. The remaining possible distributions of the critical values, as well as the case $\lambda, \mu \notin \mathbb{R}$, are studied in the same way. We summarize all this in the following

Proposition 3. Suppose that the Hamiltonian $H(x, y)$ is taken in a normal form (3) and has four distinct critical values. Then:

(i) In the real case $(0<\mu<\lambda<1)$, the continuous family of ovals $\mathcal{O}_{1}$ surrounding the center $(1,0)$ is exceptional, and the continuous family of ovals $\mathcal{O}_{\mu}$ surrounding the center $(\mu, 0)$ is not exceptional. 
(ii) In the complex case $(\lambda=\bar{\mu} \notin \mathbb{R})$, the continuous family of ovals $\mathcal{O}_{1}$ is exceptional if and only if $\lambda$ lies inside the left branch of the hyperbola $\Gamma$ (see (5) and Figure [5).

\section{THE PERIOD INTEGRAL IN A COMPLEX DOMAIN}

Assume that the level curve $\{H=h\}$ contains an oval $\delta(h)$ and consider the integral (oriented along with the vector field (4))

$$
I_{0}(h)=\oint_{\delta(h)} \frac{d x}{y}
$$

which is the derivative of the area inside $\delta(h)$ and hence determines the period of the periodic orbit lying on $\delta(h)$. Denote by $\alpha(h)$ and by $\beta(h)$ the minimal and the maximal values of $x$ for $(x, y) \in \delta(h)$. These are among the real solutions of the equation $P(x)=h$. Then one can express $I_{0}(h)$ in the form

$$
I_{0}(h)=\sqrt{2} \int_{\alpha(h)}^{\beta(h)} \frac{d x}{\sqrt{h-P(x)}} .
$$

Consider first the case $0<\mu<\lambda<1$ and assume that $\delta(h)$ belongs to either of the continuous families of ovals $\mathcal{O}_{\mu}, \mathcal{O}_{1}$ surrounding centers $(\mu, 0)$ and $(1,0)$, respectively. The corresponding families of cycles vanish respectively at $h=h_{\mu}$ and $h=h_{1}$. Denote this value in both cases by $h_{c}$ and assume that the real ovals are defined in $\Sigma=\left(h_{c}, h_{s}\right)$. Finally, denote by $x_{c}$ the $x$-coordinate of the corresponding center. We keep the same notation in the complex case and in the cases when either $\mu=0$ or $\mu=\lambda$ or $\lambda=1$. Then $x_{c}$ is the $x$-coordinate of the unique non-degenerate center (as long as $(\lambda, \mu) \notin\{(1,0),(1,1)\})$.

The integral $I_{0}(h)$ has an analytic continuation for $h<h_{s}$. We wish to prove that $I_{0}(h) \neq 0$ in $\left(-\infty, h_{c}\right)$. The precise statement is as follows.

Proposition 4. Assume that the parameters $\lambda, \mu$ in (3) are either real or lie inside the hyperbola $\Gamma$. Then the integral $I_{0}(h)$ related to any period annulus around a nondegenerate center of (4) has an analytic continuation in $\left(-\infty, h_{s}\right)$ and takes positive values there.

Proof. The claim that $I_{0}(h)$ has an analytic continuation in the interval $\left(-\infty, h_{s}\right)$ is obvious in the complex case $(\lambda=\bar{\mu} \notin \mathbb{R})$. In the real case this follows from the Dynkin diagram shown in Figure 2 For instance, if $h_{1}<h_{\mu}<h_{\lambda}<h_{0}$ as in Figure 2 and if $h_{s}=h_{\lambda}, h_{c}=h_{1}$, then the claim is obvious. If $h_{s}=h_{\lambda}, h_{c}=h_{\mu}$, then the claim is true again because the intersection number of $\delta_{\mu}$ and $\delta_{1}$ is equal to zero. The remaining four configurations of critical values and period annuli are examined in the same way (with the help of Figure 21). Note, however, that the integral $I_{0}(h)$ corresponding to the period annulus $\mathcal{O}_{e}$ (in this case $\Sigma=\left(h_{\lambda}, 0\right)$ ) has no analytic continuation on the real axis outside $\Sigma$. This is so because the singularities in both $h=h_{0}$ and $h=h_{\lambda}$ are unavoidable. The same conclusion holds for some of the degenerate cases as well.

Next we shall prove the positivity of the period integral $I_{0}(h)$ on $\left(-\infty, h_{s}\right)$. We first choose a proper formula to present $I_{0}(h)$ when $h<h_{c}$. Take $z=x+i y \in \mathbb{C}$, $h<h_{s}$ and consider the polynomial $h-P(z)$. By Taylor's formula,

$$
\begin{aligned}
h-P(z) & =h-P(x)+\frac{1}{2} y^{2} P^{\prime \prime}(x)-\frac{1}{24} y^{4} P^{\prime \prime \prime \prime}(x)-i y\left[P^{\prime}(x)-\frac{1}{6} y^{2} P^{\prime \prime \prime}(x)+\frac{1}{5} y^{4}\right] \\
& \equiv h-Q(x, y)-i y R(x, y) .
\end{aligned}
$$


Given $h<h_{s}$, consider the equation $h-P(z)=0$ and denote by $\zeta_{k}(h), k=1,2$, the two branches of the algebraic function defined by $h-P(\zeta(h)) \equiv 0$ which satisfy $\zeta\left(h_{c}\right)=\left(x_{c}, 0\right)$. These functions are unique since $P^{\prime}(z) \neq 0$ for $z \neq 0, \mu, \lambda, 1$. For $h_{c}<h<h_{s}$, one has $\zeta_{1}(h)=(\alpha(h), 0)=\alpha(h)$ and $\zeta_{2}(h)=(\beta(h), 0)=\beta(h)$ where $\alpha, \beta$ are as above. For $h<h_{c}$, one has $\zeta_{1}(h)=\bar{\zeta}_{2}(h) \in \mathbb{C}$. For a similarity in notation, we put $\zeta_{1}(h)=\alpha(h)$ and $\zeta_{2}(h)=\beta(h)=\bar{\alpha}(h)$ where $\operatorname{Im} \alpha<0$. Denote by $C_{h}$ the curve in the complex plane connecting the points $\alpha(h), \beta(h)$ along $R(x, y)=0$, if $h<h_{c}$, and along the real line $y=0$, if $h_{c}<h<h_{s}$. Then the integral $I_{0}(h)$ is expressed as

$$
I_{0}(h)=\sqrt{2} \int_{C_{h}} \frac{d z}{\sqrt{h-Q(x, y)}}, \quad h<h_{s} .
$$

Let $h<h_{c}$. We will establish below that $y=\operatorname{Im} z$ can be used as a local coordinate on $C_{h}$. Thus, $d z=\left[x^{\prime}(y)+i\right] d y$ where $x(-y)=x(y)=x$. Denote for short $y_{h}=\operatorname{Im} \beta(h)$ and write $\int_{C_{h}}=\int_{-y_{h}}^{0}+\int_{0}^{y_{h}}$. Replacing $y$ with $-y$ in the first integral, we obtain

$$
I_{0}(h)=2 \sqrt{2} \int_{0}^{y_{h}} \frac{d y}{\sqrt{Q(x(y), y)-h}}, \quad h<h_{c} .
$$

Below we study the curve $\mathcal{R}: R(x, y)=0$ and establish that on this curve the real-valued function $Q(x, y)-h$ is positive for all $h<h_{c}$.

Recall that $\mathcal{R}$ has an equation $\frac{1}{5} y^{4}-\frac{1}{6} y^{2} P^{\prime \prime \prime}(x)+P^{\prime}(x)=0$. We will study how this algebraic curve changes when varying the parameters $\lambda, \mu$. It turns out that a local analysis can be used to determine the global behavior of $\mathcal{R}$. To begin with, we observe that the Poincaré index of any sufficiently big circle $x^{2}+y^{2}=r^{2}$ subject to the vector field $d R(x, y)=0$ is -3 . The critical points of $R$ are determined from $R_{x}=R_{y}=0$, namely

(i) $y=0, P^{\prime \prime}(x)=0$;

(ii) $y^{2}=\frac{5}{12} P^{\prime \prime \prime}(x), P^{\prime \prime}(x)-\frac{5}{72} P^{\prime \prime \prime}(x) P^{\prime \prime \prime \prime}(x)=0$.

It is easy to verify that the Hessian $R_{x x} R_{y y}-R_{x y}^{2}$ is negative at any (real) nondegenerate critical point $(\xi, \eta)$. Indeed, it equals

$$
-\frac{1}{3}\left(P^{\prime \prime \prime}(\xi)\right)^{2} \quad \text { and } \quad-\frac{5}{108} P^{\prime \prime \prime}(\xi)\left(P^{\prime \prime \prime \prime}(\xi)\right)^{2}-\frac{4}{9}\left(P^{\prime \prime \prime}(\xi)\right)^{2}
$$

respectively, in cases (i) and (ii). Hence, $R$ has in general three nondegenerate saddles, and when two of the saddles collide, the resulting degenerate critical point has only hyperbolic sectors around. In particular, (ii) has no solutions for $0<$ $\mu<\lambda<1$, since all the three critical points are then given by (i). Also, in this case the curve $\mathcal{R}$ is free of critical points because the value of $R$ at the saddle $(\xi, 0)$ is $P^{\prime}(\xi) \neq 0$. In general, one can prove that $\mathcal{R}$ has no critical points outside the real line. Indeed, taking a critical point $z=\xi+i \eta$ with $\eta \neq 0$, one has $P^{\prime}(z)=R(\xi, \eta)+\eta\left(R_{y}(\xi, \eta)+i R_{x}(\xi, \eta)\right)=R(\xi, \eta) \neq 0$ unless $z=\lambda$ or $z=\mu$ (which may happen in the complex case). However, by (ii), $(\operatorname{Re} \lambda, \pm \operatorname{Im} \lambda)$ is a critical point if and only if $\lambda$ lies on the hyperbola $\Gamma$, which is not our case. We have proved that the only critical points $\mathcal{R}$ may have are $(0,0),(\lambda, 0)$ and $(1,0)$ provided that $\mu=0, \lambda=\mu$, and $\lambda=1$, respectively.

As a consequence, the branches of the level curve $\mathcal{R}$ do not intersect outside the real line and they all do escape to infinity (in the directions of $x$ and $y$ altogether, as shown by the asymptotics at infinity). This is because any closed compact curve 
necessarily surrounds a critical point having an index +1 , and $R(x, y)=$ const has no such points.

Assume first that $0<\mu<\lambda<1$. Then the four branches of $\mathcal{R}$ have no common points at all. This implies that the branches through $(0,0)$ and $(\mu, 0)$ go to $-\infty$ in the $x$-direction while the branches through $(\lambda, 0)$ and $(1,0)$ go to $+\infty$; see Figure 4 . When running any of the branches, the $y$ variable changes in a monotone way. (Otherwise, there would exist a horizontal line $y=$ const having at least 6 intersections with $\mathcal{R}$ which is impossible.) Finally, denote by $\xi$ any of $0,1, \lambda, \mu$. The local equation of $\mathcal{R}$ near the point $(\xi, 0)$ is $y^{2}=\left[6 P^{\prime \prime}(\xi) / P^{\prime \prime \prime}(\xi)\right](x-\xi)+O\left((x-\xi)^{2}\right)$, where the coefficient is negative for $\xi=0$, positive for $\xi=1$ and changes sign if $\xi=\lambda, \mu$. This latter is because $P^{\prime \prime \prime}(\lambda)$ and $P^{\prime \prime \prime}(\mu)$ change sign in $T$. On the $(\lambda, \mu)$ curve where $P^{\prime \prime \prime}$ vanishes, the local equation of $\mathcal{R}$ becomes $y^{4} \sim-5 P^{\prime \prime}(\xi)(x-\xi)$ for $\xi=\lambda, \mu$. Summing up, we obtain the form of the curve $\mathcal{R}$ as given in Figure 4 The arrows indicate the direction of the Hamiltonian vector field $d R=0$.

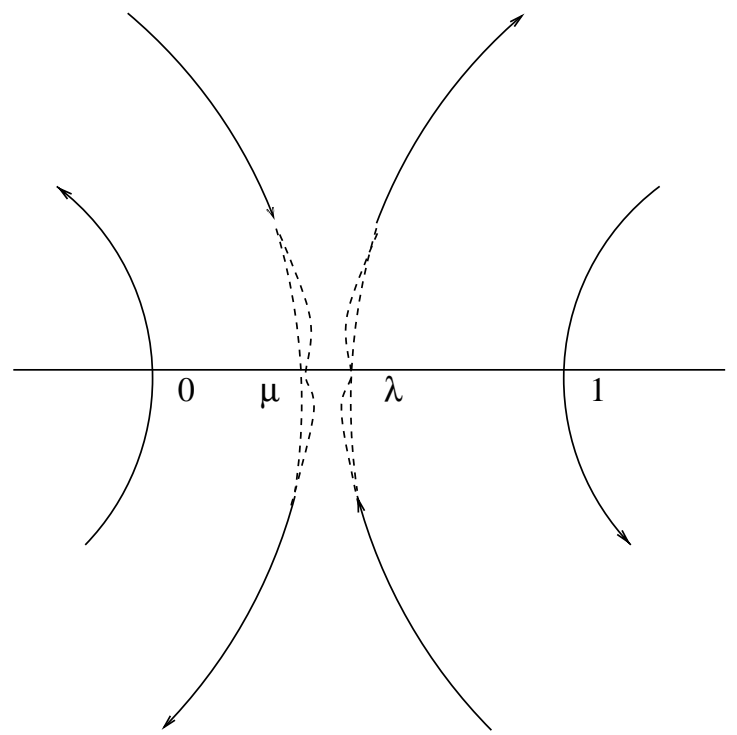

Figure 4 . The curve $R(x, y)=0$ for the case $0<\mu<\lambda<1$.

It is easy to see that $q(y)=Q(x(y), y)-h$ does not change sign when $y \in\left(0, y_{h}\right)$. As $q\left(y_{h}\right)=0$, it suffices to show that $q^{\prime}(y) \neq 0$. We first note that $\dot{y}=-R_{x}<0$ on the branch through $\left(h_{c}, 0\right)$ we consider. Then, by Cauchy-Riemann equations,

$$
Q_{x}=(y R)_{y}, \quad Q_{y}=-(y R)_{x},
$$

and we obtain on $R=0$ that

$$
q^{\prime}=Q_{y}+x^{\prime} Q_{x}=-\frac{y}{R_{x}}\left(R_{x}^{2}+R_{y}^{2}\right)<0 .
$$

This implies that $Q(x(y), y)-h>0$ for $y \in\left(0, y_{h}\right)$ and hence by (7), $I_{0}(h)>0$ for $h<h_{s}$.

The same analysis is applicable when $\mu=0$ or $\mu=\lambda$ or $\lambda=1$. The unique difference is that in Figure 4 the corresponding branches touch each other at $y=0$. 
Let us now turn to the complex case. Apart from the real case, the branches through $(0,0)$ and $(1,0)$ (let us denote them by $R_{0}$ and $R_{1}$ ) may bifurcate significantly. This is because they are not necessarily monotone with respect to $y$ and may escape to either $-\infty$ or $+\infty$, in the $x$-direction. The complex plane is divided by the hyperbola $\Gamma$ into three parts. In the outer domain $(\operatorname{Im} \lambda)^{2}>5 \operatorname{Re} \lambda(\operatorname{Re} \lambda-1)$, $R_{0}$ and $R_{1}$ behave as in Figure 4 but are not necessarily monotone in $y$. Inside the left branch of the hyperbola, $R_{0}$ and $R_{1}$ look like the branches through $\lambda$ and 1 in Figure 4 respectively. Also, inside the right branch, $R_{0}$ and $R_{1}$ behave as the branches through 0 and $\mu$ in Figure 4, respectively. All this is easily verified by a simple deformation argument, with a starting point on the appropriate side of triangle $T$.

To avoid complications, we regard only the parameters $\lambda, \mu$ in the complex plane which belong to the interior of $\Gamma$ (which is the case to be considered in our main theorem). Taking $\lambda=a+i b$, then $b^{2}<5 a(a-1)$. We are going to prove that $R_{1}$ is monotone with respect to $y$ in this domain. If so, one obtains just as in the real case that $q(y)=Q(x(y), y)-h>0$, and the proof is complete.

First consider the interior of the left branch of $\Gamma$. Then $R_{1}$ lies in the half-plane $x \geq 1$. To establish monotonicity, we rewrite $R(x, y)$ as a polynomial in $t=x-1$. One obtains

$$
\begin{aligned}
R= & t^{4}+(3-2 a) t^{3}+\left(3-4 a+a^{2}+b^{2}-2 y^{2}\right) t^{2} \\
& +\left(1-2 a+a^{2}+b^{2}+(2 a-3) y^{2}\right) t+R(1, y) .
\end{aligned}
$$

Assume that $a \leq \frac{1}{2}$. As the first two coefficients of the polynomial are positive, then according to Descartes rule of signs, (8) can have three positive roots only if the coefficient at $t^{2}$ is negative and the coefficient at $t$ is positive. However, these two conditions contradict each other. Therefore each line $y=$ const can intersect $R_{1}$ in at most two points (counting multiplicity) and hence in a single point because the number of intersections should be an odd number.

Now take $\lambda$ in the interior of the right branch of $\Gamma$ and denote by $(\xi, \eta)$ the right-most point of $R_{1}$ in the half-plane $y \geq 0$. Thus, $\xi \geq 1$ and $\eta \geq 0$. As above, one obtains with $t=\xi-x \geq 0$ that

$$
\begin{aligned}
R= & t^{4}-\frac{1}{6} P^{\prime \prime \prime \prime}(\xi) t^{3}+\left(\frac{1}{2} P^{\prime \prime \prime}(\xi)-2 y^{2}\right) t^{2}-\left(P^{\prime \prime}(\xi)-\frac{1}{6} P^{\prime \prime \prime \prime}(\xi) y^{2}\right) t \\
& +\frac{1}{5}\left(y^{2}-\frac{5}{12} P^{\prime \prime \prime}(\xi)\right)^{2} .
\end{aligned}
$$

As $\eta=0$ if $P^{\prime \prime \prime}(\xi) \leq 0$ and $\eta^{2}=\frac{5}{12} P^{\prime \prime \prime}(\xi)$ otherwise, the coefficient at $t^{2}$ is negative for $y>\eta$. Then by Descartes rule, (9) has only two positive roots and therefore the part of $R_{1}$ above the line $y=\eta$ is monotone. It remains to consider the part below it (if $\eta>0$ ). In this domain (9) can have two complex roots and Descartes rule does not yield the required result. We can proceed as follows. Assume that the part of $R_{1}$ in the upper half-plane lying below $y=\eta$ is not monotone. Denote by $\left(\xi_{1}, \eta_{1}\right)$ the first local maximum on $R_{1}$ (starting from $(1,0)$ ); thus $1<\xi_{1}<\xi$ and $0<\eta_{1}<\eta$. Also consider the branch $R_{+}$of $\mathcal{R}$ which is placed in the upper half-plane to the right of the line $x=\xi$ (and above the line $y=\eta_{1}$ ). An easy calculation yields the equations $y= \pm \sqrt{5 \pm 2 \sqrt{5}}\left(x-\frac{1+2 a}{4}\right)$ for the tangential lines of $\mathcal{R}$ at infinity. As their common point is different from $\left(\xi_{1}, \eta_{1}\right)$, there would always exist a tangential line $l$ to $R_{+}$at a finite point, which goes through $\left(\xi_{1}, \eta_{1}\right)$. However, then $l$ would intersect $R_{+}$in at least two points and $R_{1}$ in at least three 
points, which is impossible because $\mathcal{R}$ is a quartic curve. The monotonicity of $R_{1}$ is established.

Proposition 5. Assume that $\delta(h)$ is an exceptional family of ovals according to Definition 1 Then the period integral $I_{0}(h)=\int_{\delta(h)} d x / y$ has an analytic continuation in the complex domain $\mathcal{D}=\mathbb{C} \backslash\left[h_{s}, \infty\right)$ and does not vanish there.

Proof. The proof that $I_{0}(h)$ has an analytic continuation in $\mathcal{D}$ follows immediately from the definition of an exceptional family of ovals. To count the zeros of the analytic function $I_{0}(h)$ in $\mathcal{D}$, we apply the argument principle to the complex domain $\mathcal{D}_{r}$ obtained from $\mathcal{D}$ by removing the discs $\left\{h \in \mathbb{C}:\left|h-h_{s}\right|<r\right\},\{h \in \mathbb{C}:|h|>1 / r\}$, where $r>0$ is a small enough constant. For this purpose we evaluate the increase of the argument of $I_{0}(h)$ when $h$ runs the boundary of $\mathcal{D}_{r}$ in a positive direction.

We first consider the real case $(0<\mu<\lambda<1)$. The Picard-Lefschetz formula implies that in a suitable complex neighborhood of $h_{s}$ it holds

$$
I_{0}(h)=\varphi(h) \log \left(h-h_{s}\right)+\psi(h), \quad \varphi(h)=\frac{1}{2 \pi} \int_{\delta_{s}(h)} \frac{d x}{y},
$$

where $\varphi, \psi$ are locally holomorphic and, as is easily checked, $\varphi\left(h_{s}\right) \neq 0$.

The behavior of $I_{0}(h)$ for $|h|$ sufficiently big follows from the Picard-Lefschetz formula too, but the argument is more delicate. Let $l$ be a simple closed path which makes one turn in a positive direction around all the critical values of $H$. It induces a monodromy map

$$
l_{*}: H_{1}\left(\Gamma_{\tilde{h}}, \mathbb{Z}\right) \rightarrow H_{1}\left(\Gamma_{\tilde{h}}, \mathbb{Z}\right), \quad \Gamma_{\tilde{h}}=\{(x, y) \in \mathbb{C}: H(x, y)=\tilde{h}\} .
$$

It is shown for instance in 12 that $l_{*}$ coincides with the operator of classical monodromy of the singularity $\frac{1}{2} y^{2}+\frac{1}{5} x^{5}$ (which is the highest weighted homogeneous part of $H)$. The characteristic polynomial $P(z)$ of $l_{*}$ is now easily computed to be equal to $P(z)=\left(z^{5}+1\right) /(z+1)$ (see [9]). This shows that in a neighborhood of $h=\infty$ on the projective sphere the integral $I_{0}(h)$ is a meromorphic function of $h^{1 / 10}$. The substitution

$$
x \rightarrow x h^{1 / 5}, \quad y \rightarrow y h^{1 / 2}, \quad h \rightarrow \infty
$$

defines an isotopy of the regular fibers of $H$ and the regular fibers of its weighted homogeneous part $\frac{1}{2} y^{2}+\frac{1}{5} x^{5}[12$. From this fact we deduce that

$$
I_{0}(h)=c_{\infty} h^{1 / 5-1 / 2}\left(1+O\left(h^{-1 / 10}\right)\right) .
$$

For a further use we note that $c_{\infty} \neq 0$. Indeed, the one-form $d x / y$ is not cohomologous to zero on the regular fibers of the polynomial $\frac{1}{2} y^{2}+\frac{1}{5} x^{5}$ (this follows from [13, Theorem 1.1]). On the other hand, the form of the characteristic polynomial of $l_{*}$ shows that it is irreducible over the field of rational numbers. Therefore the span of $l_{*}^{k} \delta, k=0,1,2,3$, generates the whole homology group of the fiber. This implies that if $c_{\infty}=0$, then the restriction of $d x / y$ on the regular fibers of $\frac{1}{2} y^{2}+\frac{1}{5} x^{5}$ defines the zero cohomology class which is a contradiction.

The above consideration shows that the decrease of the argument of $I_{0}(h)$ along $\{h \in \mathbb{C}:|h|=1 / r\}$ is close to $3 \pi / 5$ and the increase of the argument of $I_{0}(h)$ along $\left\{h \in \mathbb{C}:\left|h-h_{s}\right|=r\right\}$ is close to zero.

We claim further that the imaginary part of $I_{0}(h)$ along $\left(r+h_{s}, 1 / r\right)$ does not vanish. Namely, denote the two determinations of $I_{0}(h)$ on $\left(r+h_{s}, 1 / r\right)$ by $I_{0}^{ \pm}(h)$. 
Similarly, denote by $\delta^{ \pm}(h)$ the two determinations of the continuous family $\delta(h)$ on $\left(r+h_{s}, 1 / r\right)$. Since $I_{0}$ is a real analytic function along $\left(-\infty, h_{s}\right)$, we have

$$
I_{0}^{+}(h)=\overline{I_{0}^{-}(h)}, \quad \delta^{+}(h)=\overline{\delta^{-}(h)}, \quad h>h_{s},
$$

and hence $2 \sqrt{-1} \operatorname{Im} I_{0}^{+}(h)=I_{0}^{+}(h)-I_{0}^{-}(h)$. On the other hand $\delta(h)$ is an exceptional family of ovals. Therefore the Picard-Lefschetz formula implies that for $h>h_{s}$,

$$
\delta^{+}(h)-\delta^{-}(h)=\gamma(h), \quad I_{0}^{+}(h)-I_{0}^{-}(h)=\int_{\gamma(h)} \frac{d x}{y},
$$

where $\gamma(h)$ is a continuous family of cycles vanishing at $h=h_{s}$, with intersection number $\left\langle\gamma, \delta^{-}\right\rangle=1$.

Finally, we note that after substituting $y$ by $\sqrt{-1} y$, the family of cycles $\gamma(h)$ corresponds to a family of ovals surrounding a center. Moreover, in the real case the full bifurcation diagram (consisting of the boundary of $T$ and the parts of the curves $h_{\lambda}=h_{0}, h_{\mu}=h_{1}, h_{0}=h_{1}$ inside) images onto itself. For later use we also mention that in the complex case, the interior of the hyperbola $\Gamma$ images onto itself. All this holds because the corresponding Hamiltonians when taken in a normal form (3) are related by the formula

$$
H(x, \sqrt{-1} y, \lambda, \mu)=h_{1}-H(1-x, y, 1-\mu, 1-\lambda) .
$$

Hence, it follows from Proposition 4 that $\int_{\gamma(h)} d x / y$ does not vanish along $\left(h_{s}, \infty\right)$. Therefore if $h \in\left(r+h_{s}, 1 / r\right)$, then $\operatorname{Im} I_{0}^{ \pm}(h) \neq 0$ and hence the increase of the argument of $\operatorname{Im} I_{0}^{ \pm}(h)$ along $\left(r+h_{s}, 1 / r\right)$ is at most $\pi$.

Summing up the above information we conclude that the increase of the argument of $I_{0}(h)$ along the boundary of $\mathcal{D}_{r}$ is strictly less than $2 \pi$. By the argument principle, $I_{0}(h)$ does not vanish in $\mathcal{D}_{r}$, and hence in $\mathcal{D}$.

The complex case when $\lambda=\bar{\mu} \notin \mathbb{R}$ is studied in the same way.

\section{The Chebyshev Property}

Suppose that the real polynomial $H=y^{2}+P(x)$ has distinct critical values. Let $\delta(h) \subset\{H=h\}$ be a continuous family of ovals defined on a maximal open interval $\Sigma=\left(h_{c}, h_{s}\right)$, where for $h=h_{c}$ the oval degenerates to a point $\delta\left(h_{c}\right)$ which is a center and for $h=h_{s}$ the oval becomes a homoclinic loop of the Hamiltonian system $d H=0$. Denote $\mathcal{D}=\mathbb{C} \backslash\left[h_{s}, \infty\right)$. The following theorem is our main result.

Theorem 1. Let $\delta(h), h \in \Sigma$, be an exceptional family of ovals of (3). Then the real vector space $\mathcal{A}=\left\{\alpha_{0} I_{0}+\alpha_{1} I_{1}: \alpha_{0}, \alpha_{1} \in \mathbb{R}\right\}$ of Abelian integrals

$$
I_{0}(h)=\int_{\delta(h)} \frac{d x}{y}, \quad I_{1}(h)=\int_{\delta(h)} \frac{x d x}{y}, \quad h \in \Sigma,
$$

is Chebyshev in $\mathcal{D}$ and hence in $\Sigma$.

Proof. Let $\delta(h)$, where $h \in \Sigma=\left(h_{c}, h_{s}\right)$, be an exceptional family of ovals. According to Proposition [5, it suffices to show that the analytic function $F(h)=$ $\alpha_{0}+I_{1}(h) / I_{0}(h)$ has at most one zero in the domain $\mathcal{D}$. To prove this claim we apply the argument principle to $F(h)$ in the complex domain $\mathcal{D}_{r}$ obtained from $\mathcal{D}$ by removing the discs $\left\{h \in \mathbb{C}:\left|h-h_{s}\right|<r\right\},\{h \in \mathbb{C}:|h|>1 / r\}$, where $r>0$ is a small enough constant. 
As in the proof of Proposition [5, we may check that:

- if $h \sim \infty$, then $I_{1}(h)$ is meromorphic with respect to $h^{-1 / 10}$ and

$$
I_{1}(h)=c_{\infty} h^{2 / 5-1 / 2}\left(1+O\left(h^{-1 / 10}\right)\right) \text { where } c_{\infty} \neq 0 ;
$$

- if $h \sim h_{s}$, then

$$
I_{1}(h)=\varphi_{1}(h) \log \left(h-h_{s}\right)+\psi_{1}(h), \quad \varphi_{1}(h)=\frac{1}{2 \pi} \int_{\delta_{s}(h)} \frac{x d x}{y},
$$

where $\varphi_{1}$ and $\psi_{1}$ are locally holomorphic. Moreover, it is easily verified that $\lim _{h \rightarrow h} I_{1}(h) / I_{0}(h)=\varphi_{1}\left(h_{s}\right) / \varphi\left(h_{s}\right)=x_{s}$, where $\left(x_{s}, 0\right)$ is the saddle point corresponding to the critical value $h_{s}$.

Therefore the increase of the argument of $F(h)$ along $\{h \in \mathbb{C}:|h|=1 / r\}$ is close to $2 \pi / 5$ and the increase of the argument of $F(h)$ along $\{h \in \mathbb{C}:|h|=r\}$ is close to zero or strictly negative.

We claim further that

- the imaginary part of $F(h)$ along $\left(r+h_{s}, 1 / r\right)$ does not vanish.

If this claim were proved, then summing up the above information we would get that the argument of $F(h)$ increases along the boundary of $\mathcal{D}_{r}$ by at most $2 \pi+2 \pi / 5$. Thus the function $F(h)$ can have at most one zero in $\mathcal{D}_{r}$ and hence in $\mathbb{C} \backslash\left[h_{s}, \infty\right)$.

It remains to prove the above claim. Recall that, since $\delta(h)$ is an exceptional family of ovals, then the two determinations of $F(h)$ over $\left(h_{s}, \infty\right)$, namely $F^{ \pm}(h)$, are analytic along $\left(h_{s}, \infty\right)$. The function $F(h)$ is real analytic along $\left(-\infty, h_{s}\right)$ and hence

$$
2 \sqrt{-1} \operatorname{Im} F(h)=F^{+}(h)-F^{-}(h) \forall h \in\left(h_{s}, \infty\right) .
$$

Denote by $\delta^{ \pm}(h)$ the two determinations of $\delta(h), h \in \mathcal{D}$ on $\left(h_{s}, \infty\right)$, and let

$$
\Gamma_{h}=\left\{(x, y) \in \mathbb{C}^{2}: H(x, y)=h\right\} .
$$

It was shown in the proof of Proposition 5 that

$$
\int_{\delta^{ \pm}(h)} \frac{d x}{y} \neq 0 \quad \forall h \in\left(h_{s}, \infty\right) .
$$

Then we obtain for each $h \in\left(h_{s}, \infty\right)$

$$
2 \sqrt{-1} \operatorname{Im} F(h)=\frac{\int_{\delta^{+}(h)} \frac{x d x}{y}}{\int_{\delta^{+}(h)} \frac{d x}{y}}-\frac{\int_{\delta^{-}(h)} \frac{x d x}{y}}{\int_{\delta^{-}(h)} \frac{d x}{y}}=\frac{\operatorname{det}\left(\begin{array}{cc}
\int_{\delta^{+}(h)} \frac{x d x}{y} & \int_{\delta^{+}(h)} \frac{d x}{y} \\
\int_{\delta^{-}(h)} \frac{x d x}{y} & \int_{\delta^{-}(h)} \frac{d x}{y}
\end{array}\right)}{\left|\int_{\delta^{ \pm}(h)} \frac{d x}{y}\right|^{2}} .
$$

Therefore we have to prove that the analytic function

$$
\Delta(h)=\operatorname{det}\left(\begin{array}{ll}
\int_{\delta^{+}(h)} \frac{x d x}{y} & \int_{\delta^{+}(h)} \frac{d x}{y} \\
\int_{\delta^{-}(h)} \frac{x d x}{y} & \int_{\delta^{-}(h)} \frac{d x}{y}
\end{array}\right), \quad h \in\left(h_{s}, \infty\right),
$$

does not vanish.

Suppose first that $h=h_{0} \in\left(h_{s}, \infty\right)$ is a critical value of $H$. The affine curve $\Gamma_{h_{0}}$ is singular of arithmetic genus two. Let $\bar{\Gamma}_{h_{0}}$ be the corresponding completed and normalized Riemann surface. As the geometric genus of $\bar{\Gamma}_{h_{0}}$ is one and the intersection number $\left\langle\delta^{+}, \delta^{-}\right\rangle$equals +1 , then $\delta^{+}\left(h_{0}\right)$ and $\delta^{-}\left(h_{0}\right)$ form a canonical homology basis of $\bar{\Gamma}_{h_{0}}$. We have $P(x)=\left(x-x_{0}\right)^{2}\left(x-x_{1}\right)\left(x-x_{2}\right)\left(x-x_{3}\right)$, where $x_{i} \neq x_{j}$ for $i \neq j$. Therefore, $\left(x-x_{0}\right) d x / y$ induces a holomorphic one-form on $\bar{\Gamma}_{h_{0}}$ and $d x / y$ induces a meromorphic one-form of the third kind on $\bar{\Gamma}_{h_{0}}$ with simple poles 
at $P^{ \pm}$, where $P^{ \pm}$are the two distinct pre-images of the singular point $\left(x_{0}, 0\right) \in \Gamma_{h_{0}}$ under the canonical projection $\bar{\Gamma}_{h_{0}} \rightarrow \Gamma_{h_{0}}$. The reciprocity law for differentials of the first and third kind [18] applied to $\left(x-x_{0}\right) d x / y, d x / y$ on the elliptic curve $\bar{\Gamma}_{h_{0}}$ gives

$$
\begin{aligned}
\Delta(h) & =2 \pi \sqrt{-1}\left[\left.\operatorname{Res}\right|_{P^{+}}\left(\frac{d x}{y} \int_{P^{0}}^{P} \frac{\left(x-x_{0}\right) d x}{y}\right)+\left.\operatorname{Res}\right|_{P^{-}}\left(\frac{d x}{y} \int_{P^{0}}^{P} \frac{\left(x-x_{0}\right) d x}{y}\right)\right] \\
& =\frac{ \pm 2 \pi \sqrt{-1}}{\sqrt{\left(x_{0}-x_{1}\right)\left(x_{0}-x_{2}\right)\left(x_{0}-x_{3}\right)}} \int_{P^{-}}^{P^{+}} \frac{d x}{\sqrt{\left(x-x_{1}\right)\left(x-x_{2}\right)\left(x-x_{3}\right)}}
\end{aligned}
$$

where the path of integration is on the Riemann surface $\bar{\Gamma}_{h_{0}}$ of the affine elliptic curve

$$
\left\{y^{2}=\left(x-x_{1}\right)\left(x-x_{2}\right)\left(x-x_{3}\right)\right\}
$$

and $P^{ \pm}=\left(x_{0}, \pm \sqrt{\left(x_{0}-x_{1}\right)\left(x_{0}-x_{2}\right)\left(x_{0}-x_{3}\right)}\right)$. The function

$$
P \rightarrow \int^{P} \frac{d x}{\sqrt{\left(x-x_{1}\right)\left(x-x_{2}\right)\left(x-x_{3}\right)}}
$$

is a uniformizing variable on the elliptic curve $\bar{\Gamma}_{h_{0}}$ which shows that

$$
\int_{P^{-}}^{P^{+}} \frac{d x}{\sqrt{\left(x-x_{1}\right)\left(x-x_{2}\right)\left(x-x_{3}\right)}}=0 \Rightarrow P^{+}=P^{-} .
$$

As $P^{+} \neq P^{-}$, then $\Delta\left(h_{0}\right) \neq 0$.

Suppose now that $h \in\left(h_{s}, \infty\right)$ is a regular value of $H$. The intersection number $\left\langle\delta^{+}, \delta^{-}\right\rangle$equals +1 and it is easy to check that we may choose a canonical homology basis $\delta_{1}(h), \ldots, \delta_{4}(h)$ of the lattice $H_{1}\left(\bar{\Gamma}_{h}, \mathbb{Z}\right)$ such that $\delta_{1}(h)=\delta^{+}, \delta_{3}(h)=\delta^{-}$. Further, let $\omega_{1}, \omega_{2}$ be a normalized basis of holomorphic differentials on $\bar{\Gamma}_{h}$. The corresponding period lattice $\Pi$ reads

$$
\Pi=\left(\begin{array}{cccc}
\int_{\delta_{1}(h)} \omega_{1} & \int_{\delta_{2}(h)} \omega_{1} & \int_{\delta_{3}(h)} \omega_{1} & \int_{\delta_{4}(h)} \omega_{1} \\
\int_{\delta_{1}(h)} \omega_{2} & \int_{\delta_{2}(h)} \omega_{2} & \int_{\delta_{3}(h)} \omega_{2} & \int_{\delta_{4}(h)} \omega_{2}
\end{array}\right)=\left(\begin{array}{cccc}
1 & 0 & a & b \\
0 & 1 & c & d
\end{array}\right),
$$

where

$$
\left(\begin{array}{c}
\omega_{1} \\
\omega_{2}
\end{array}\right)=\left(\begin{array}{ccc}
\int_{\delta_{1}(h)} \frac{x d x}{y} & \int_{\delta_{2}(h)} \frac{x d x}{y} \\
\int_{\delta_{1}(h)} \frac{d x}{y} & \int_{\delta_{2}(h)} \frac{d x}{y}
\end{array}\right)^{-1}\left(\begin{array}{c}
\frac{x d x}{y} \\
\frac{d x}{y}
\end{array}\right) .
$$

We get that

$$
\Delta(h)=\operatorname{det}\left(\begin{array}{cc}
\int_{\delta_{1}(h)} \frac{x d x}{y} & \int_{\delta_{2}(h)} \frac{x d x}{y} \\
\int_{\delta_{1}(h)} \frac{d x}{y} & \int_{\delta_{2}(h)} \frac{d x}{y}
\end{array}\right) \operatorname{det}\left(\begin{array}{cc}
1 & a \\
0 & c
\end{array}\right)
$$

and hence

$$
\Delta(h)=0 \Leftrightarrow c=0 .
$$

Let $\Lambda$ be the lattice generated by the columns of $\Pi$. The Riemann bilinear relations [18 on $\Pi$ imply

$$
b=c .
$$

Therefore if $\Delta(h)=0$, then the Jacobian variety $J\left(\bar{\Gamma}_{h}\right)=\mathbb{C}^{2} / \Lambda$ is a direct product of the two elliptic curves

$$
\mathbb{C} /\{\mathbb{Z} \oplus a \mathbb{Z}\}, \quad \mathbb{C} /\{\mathbb{Z} \oplus d \mathbb{Z}\} .
$$


It is well known that this is impossible (e.g. the Remark on page 49 in [2]) which completes the proof of Theorem 1 .

For convenience of the reader, we present a proof of the last claim. Consider the Riemann theta function

$$
\theta(z)=\sum_{m \in \mathbb{Z}^{2}} e^{\pi \sqrt{-1}\langle m, B m\rangle+2 \pi \sqrt{-1}\langle m, z\rangle}
$$

and the corresponding theta divisor $\Theta=\left\{z \in J\left(\bar{\Gamma}_{h}\right): \theta(z)=0\right\}$, where

$$
B=\left(\begin{array}{ll}
a & b \\
c & d
\end{array}\right), \quad z=\left(\begin{array}{l}
z_{1} \\
z_{2}
\end{array}\right), \quad\langle m, z\rangle=m_{1} z_{1}+m_{2} z_{2} .
$$

By the Riemann theorem, the divisor $\Theta$ is isomorphic to the curve $\bar{\Gamma}_{h}$ and in particular is irreducible. If $\Delta(h)=0$, then $b=c=0$ and $\theta(z)$ factorizes into a product of two elliptic theta functions

$$
\theta(z)=\sum_{m_{1} \in \mathbb{Z}} e^{\pi \sqrt{-1} a m_{1}^{2}+2 \pi \sqrt{-1} m_{1} z_{1}} \sum_{m_{2} \in \mathbb{Z}} e^{\pi \sqrt{-1} d m_{2}^{2}+2 \pi \sqrt{-1} m_{2} z_{2}} .
$$

Therefore the Riemann theta divisor $\Theta$ is a product of the two elliptic curves (10) which is a contradiction.

\section{Concluding Remarks}

We finish this paper with some general hypotheses concerning the zeros of the function $\alpha_{0} I_{0}+\alpha_{1} I_{1}$. In the previous section we demonstrated that this linear combination belongs to a Chebyshev space, provided that the continuous family of ovals $\{\delta(h)\}$ is exceptional. In the non-exceptional case a big part of our proof still holds, provided that $\lambda$ and $\mu$ are real. This fact combined with the exact local result obtained in Proposition 1 suggests the following more general statement.

Conjecture 1. Assume that the polynomial $P(x)$ has distinct real critical values. Then the corresponding vector space of Abelian integrals $\alpha_{0} I_{0}+\alpha_{1} I_{1}$ is Chebyshev with accuracy at most one in $\mathcal{D}$.

One may also ask whether the upper bound (two) for the number of the zeros near the center always holds in the whole interval $\Sigma$ and next, whether the bounds for the real zeros when $h \in \Sigma$ and for the complex zeros when $h$ belongs to a certain complex extension of $\Sigma$ do coincide. It turns out that the answers to all these questions are negative. In particular, we establish below that when $P(x)$ has complex critical values, then there are three zeros in $\Sigma$ for appropriate $\lambda \in \mathbb{C}$.

One can try to predict the possible number of real zeros by studying the behaviour of the ratio $F(h)=I_{1}(h) / I_{0}(h)$ near the ends of the interval $\Sigma$. For reader's convenience, we first recall some formulas.

Let us take a continuous function $f(x)$ and consider the integral

$$
I(h)=\int_{\delta(h)} \frac{f(x) d x}{y}, \quad h \in \Sigma,
$$

where $\Sigma=\left(h_{c}, h_{s}\right)$ for the families $\mathcal{O}_{1}, \mathcal{O}_{\mu}$ and $\Sigma=\left(h_{s}, h_{0}\right)$ for the family $\mathcal{O}_{e}$. Using the Picard-Lefschetz formula or direct calculations of integrals, one can obtain the following asymptotical expansions in a neighbourhood of the critical levels $h_{s}$ corresponding to a saddle or a cuspidal point $\left(x_{s}, 0\right)$. 
(i) For the saddle-loop and eight-loop cases,

$$
I(h)=-c_{s} f\left(x_{s}\right) \log \left|h-h_{s}\right|+\varphi(h),
$$

where $c_{s}$ is a specific positive constant independent on $f$ and $\varphi(h)$ is a continuous function in a neighbourhood of $h_{s}$. We are not going to write up the exact expression of $c_{s}$ since we do not need it here.

(ii) For the heteroclinic loop cases,

$$
I(h)=-\left(c_{0} f(0)+c_{s} f\left(x_{s}\right)\right) \log \left|h-h_{s}\right|+\varphi(h),
$$

where $c_{0}, c_{s}$ and $\varphi(h)$ are as above.

(iii) For the cuspidal loop cases,

$$
I(h)=c_{s} f\left(x_{s}\right)\left|h-h_{s}\right|^{-1 / 6}+\varphi(h),
$$

where $c_{s}$ and $\varphi(h)$ are as above.

(In fact, these formulas hold in a much more general context.) Applying the above expansions to $F(h)$, we get $F\left(h_{s}\right)=x_{s} /\left(1+c_{0} / c_{s}\right)$ for the heteroclinic loop case and $F\left(h_{s}\right)=x_{s}$ for all the remaining cases. Moreover, concerning the saddleloops, it is evident that $F(h)-x_{s} \neq 0$ in $\Sigma$ and the sign of the expression depends on the position of the saddle with respect to the family of ovals. Now consider the case of $\mathcal{O}_{e}$, where the situation is more complicated. For $h=h_{\lambda}$, we obtain from (3) that

$$
y^{2}=\frac{2}{5}(x-\lambda)^{2} p(x), \quad p(x)=\left(x-x_{1}\right)\left(x-x_{2}\right)\left(x_{3}-x\right),
$$

where $x_{1}<0<x_{2} \leq \lambda \leq x_{3}$ and $x_{2}, x_{3}$ are the left-most and the right-most points of the eight-loop (or the cuspidal loop, as a limit case). By (i)-(iii), the function $\varphi(h)=I_{1}(h)-\lambda I_{0}(h)$ is continuous in a neighbourhood of $h_{\lambda}$, which immediately yields that

$$
\varphi\left(h_{\lambda}\right)=\sqrt{10}\left(\int_{\lambda}^{x_{3}}-\int_{x_{2}}^{\lambda}\right) \frac{d x}{\sqrt{p(x)}} .
$$

Clearly, $\varphi\left(h_{\lambda}\right) \rightarrow-\infty$ when $x_{2} \rightarrow x_{1}$, and $\varphi\left(h_{\lambda}\right) \rightarrow \pm \sqrt{10} \int_{x_{2}}^{x_{3}} d x / \sqrt{p(x)}$ when $\lambda \rightarrow x_{2}$ and $\lambda \rightarrow x_{3}$, respectively. As $x_{2}=x_{1}$ is equivalent to $h_{\lambda}=0, \lambda=x_{2}$ is equivalent to $\lambda=\mu$ and $\lambda=x_{3}$ is equivalent to $\lambda=1$, this means that the curve

$$
\gamma_{s}=\left\{(\lambda, \mu) \in T: I_{1}\left(h_{\lambda}\right)-\lambda I_{0}\left(h_{\lambda}\right)=0\right\}
$$

connects the points $(0,0)$ and $(1,1)$ and lies in the part of $T$ above $\gamma$. Surprisingly, although expressed in terms of incomplete elliptic integrals, it turns out that $\gamma_{s}$ is a part of an algebraic curve of degree 6 . This can be verified by transforming the integrals to a standard form. From the explicit algebraic equation thus obtained it is easily seen that $\gamma_{s}$ is a simple and connected curve (we omit the details). The curve $\gamma_{s}$ is an element of the bifurcation diagram because for $h$ close to $h_{\lambda}$, the function $F(h)-\lambda$ will change the sign when crossing $\gamma_{s}$ in the parameter space.

Among other things, the above analysis implies that for $\lambda=\mu$, the function $\alpha_{0} I_{0}+\alpha_{1} I_{1}=I_{0}\left(\alpha_{0}+\alpha_{1} F\right)$ can have three zeros in $\left(h_{1}, h_{\lambda}\right) \cup\left(h_{\lambda}, h_{0}\right)$. Hence, the same fact remains true for $\mathcal{O}_{1}$ and the respective $\Sigma=\left(h_{1}, h_{0}\right)$ in the complex case, at least for parameters $\lambda=a+i b$ with $a \in(0,1)$ and $|b|$ small. Based on the behaviour of $F$ near the other endpoint which corresponds to a center (see below), we conjecture that the domain in the parameter space $\lambda \in \mathbb{C}$, where the equation $F(h)=$ const can have three real zeros, is surrounded by a curve on which they join into a triple zero. 


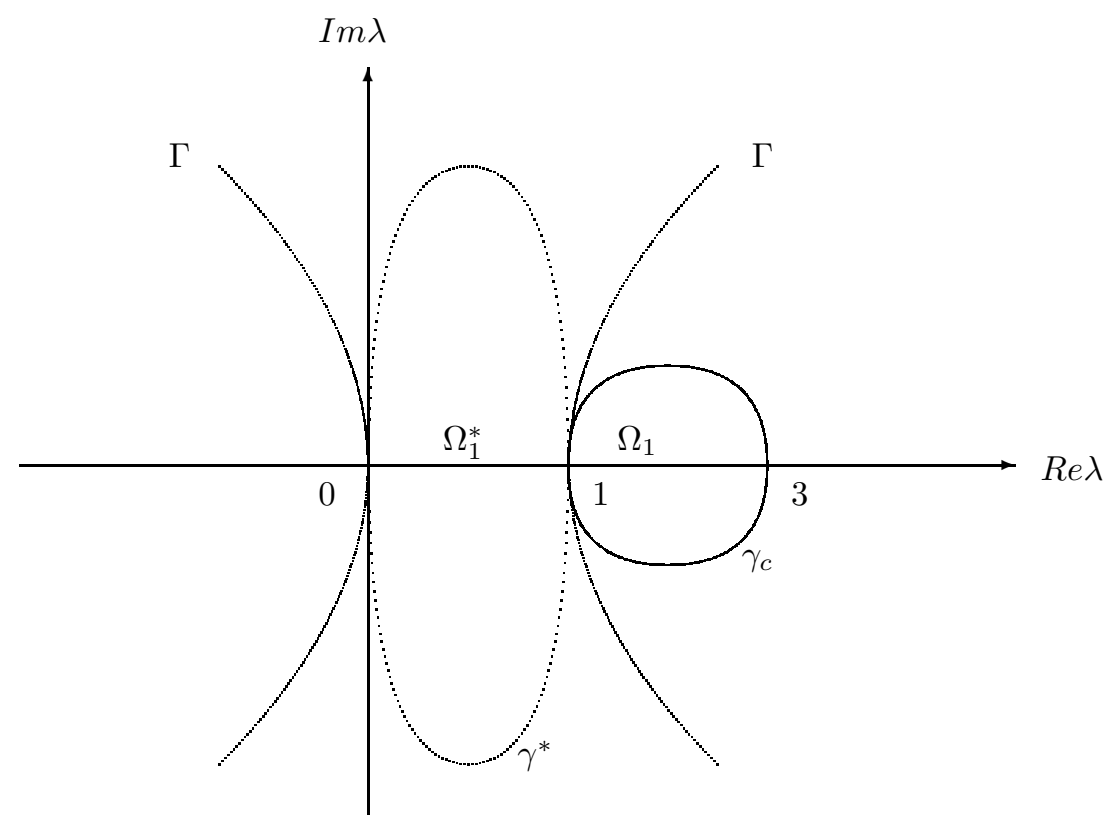

Figure 5. Partition of the parameter space in the complex case $\lambda=\bar{\mu} \in \mathbb{C}$.

Looking at the bifurcation diagram again, let us denote by $\gamma_{c}$ the curve

$$
\begin{aligned}
& \gamma_{c}=\left\{(\lambda, \mu) \in T: \lambda=\frac{3 \mu^{2}-2 \mu}{2 \mu-1}\right\} \quad \text { (in the real case), } \\
& \gamma_{c}=\{\lambda \in \mathbb{C}:|\lambda-2|=1\} \quad \text { (in the complex case). }
\end{aligned}
$$

Further, let us denote by $\gamma^{*}$ the (hypothetical) curve

$$
\gamma^{*}=\left\{\lambda \in \mathbb{C}: \exists h^{*} \in \Sigma \text { with } F^{\prime}\left(h^{*}\right)=F^{\prime \prime}\left(h^{*}\right)=0\right\} .
$$

We believe that $\gamma^{*}$ is a simple closed curve in the complex plane going through $(0,0)$ and $(1,0)$. On $\gamma^{*}$, a bifurcation of a triple real zero into a simple one occurs.

Next, in the real case, denote by $\Omega_{\mu}$ the part of $T$ placed between the curves $\gamma_{c}$ and $\gamma$, by $\Omega_{e}$ the part of $T$ placed between the curve $\gamma_{s}$ and the line $\lambda=\mu$, and finally, in the complex case, denote by $\Omega_{1}$ the unit disk inside $\gamma_{c}$ and by $\Omega_{1}^{*}$ the interior of $\gamma^{*}$ (see Figures 5 and 6).

Proposition 6. The vector spaces $\mathcal{A}_{\mu}, \mathcal{A}_{e}, \mathcal{A}_{1}$ of Abelian integrals $\alpha_{0} I_{0}+\alpha_{1} I_{1}$ corresponding to the continuous families of ovals $\mathcal{O}_{\mu}$ for $(\lambda, \mu) \in \Omega_{\mu}, \mathcal{O}_{e}$ for $(\lambda, \mu) \in$ $\Omega_{e}$, and $\mathcal{O}_{1}$ for $\lambda \in \Omega_{1} \cup \Omega_{1}^{*}$, are not Chebyshev in the respective intervals $\Sigma$.

Proof. The assertion follows from local analysis. Consider first $\mathcal{O}_{\mu}$. Then $\Sigma=$ $\left(h_{\mu}, 0\right)$ and $F\left(h_{\mu}\right)=\mu>0, F(0)=0$. By the proof of Proposition 1, we obtain that $F^{\prime}\left(h_{\mu}\right)>0$ is equivalent to $a_{1}<0$. This coefficient is easily calculated from the normal form (3) and the result is

$$
a_{1}=\frac{P^{\prime \prime \prime}(\mu)}{3 P^{\prime \prime}(\mu)}=\frac{6 \mu^{2}-4 \lambda \mu-4 \mu+2 \lambda}{3 \mu(\lambda-\mu)(1-\mu)}
$$

which proves the claim. The proof in the case of $\mathcal{O}_{1}$ is similar. The assertion for $\mathcal{O}_{e}$ follows from the definition of $\gamma_{s}$. 


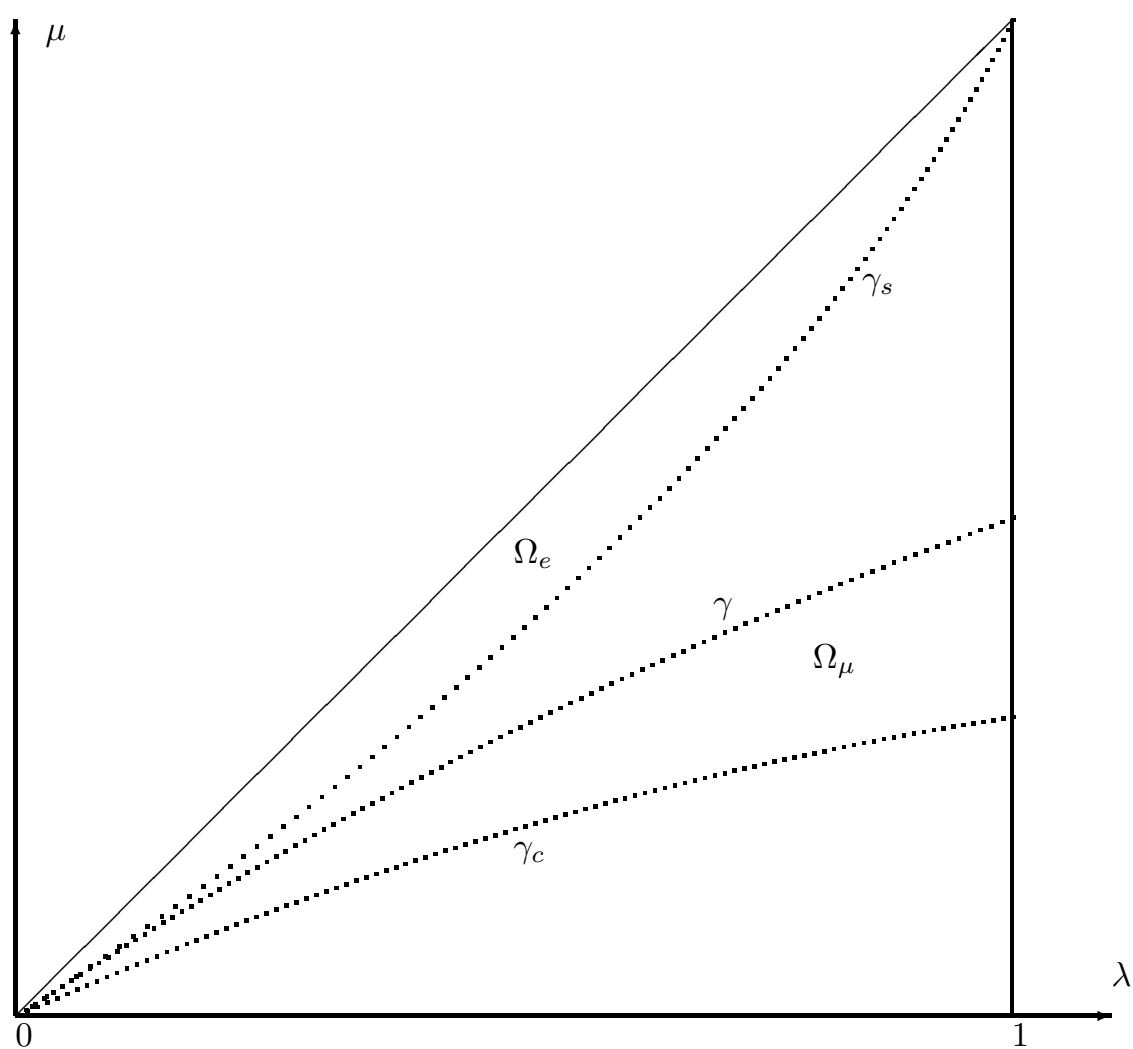

Figure 6. Partition of the parameter space in the real case $\lambda, \mu \in$ $\mathbb{R}$.

Making use of all of the information about the local behaviour of $F$ near the endpoints of $\Sigma$, one can formulate the following hypothesis.

Conjecture 2. (i) The vector spaces $\mathcal{A}_{\mu}, \mathcal{A}_{e}$ are Chebyshev with accuracy one in $\Sigma$ for the cases considered in Proposition [6, and they are Chebyshev in $\Sigma$ for all the remaining cases.

(ii) The vector space $\mathcal{A}_{1}$ is Chebyshev with accuracy one in $\Sigma$ for $\lambda \in \Omega_{1}$, it is Chebyshev with accuracy two in $\Sigma$ for $\lambda \in \Omega_{1}^{*}$ and it is Chebyshev in $\Sigma$ for the remaining cases.

(iii) Given $\alpha_{0}$ and $\alpha_{1}$, the total number of zeros of the function $\alpha_{0} I_{0}+\alpha_{1} I_{1}$ relative to all the period annuli is three and this bound is attained in $\Omega_{e} \cup \Omega_{1}^{*}$.

It is plausible that Conjecture 2 is a consequence of Conjecture 1, except in the case when the polynomial $P(x)$ has complex critical points.

In a more general context, let $P(x)$ be a real polynomial of degree $n \geq 5$ and $H=y^{2}+P(x)$ be the corresponding hyperelliptic Hamiltonian. It would also be interesting to study the linear space $\mathcal{A}_{P}$ spanned by integrals of the whole range of independent differentials $x^{k} d x / y, 0 \leq k \leq n-2$ and not only of the holomorphic ones (when $k \leq g-1$ with $g=\left[\frac{1}{2}(n-1)\right]$ ); even more generally, one can consider the $\mathbb{R}$-linear space $\mathcal{A}_{P, d}$ of integrals from all polynomial one-forms of a given degree 
$d \geq g$. The latter has a dimension $\operatorname{dim} \mathcal{A}_{P, d}=\left[\frac{1}{2} d(n-1)-\frac{1}{4}(n-2)^{2}+\frac{1}{2}\right]$ (see, e.g., 13]) and can be expressed by the sum

$$
\sum_{k=0}^{n-2} p_{k}(t) \int_{\delta(t)} x^{k} y d x, \quad p_{k} \in \mathbb{R}[t], \quad \operatorname{deg} p_{k} \leq \frac{d-k-1}{2} .
$$

According to a general conjecture [20], one can expect that $\mathcal{A}_{P}$ and $\mathcal{A}_{P, d}$ would be Chebyshev with accuracy determined by the degree $n$ of the polynomial $P$. Compared to the problem considered in the present paper, the following more difficult problems could also be studied:

- the space of Abelian integrals of the first kind related to genus-three hyperelliptic curves $(\operatorname{deg} P=7,8)$. It is a vector space of dimension three;

- the space $\mathcal{A}_{P}, \operatorname{deg} P=5,6$. It is a free $\mathbb{R}[t]$ module with four, respectively five, generators.

We hope that these problems can be settled by combining the methods of the present paper with deformation arguments as in [16. Properly understood, this might lead to the solution of the problem for arbitrary degrees $n$ and $d$.

\section{ACKNOWLEDGMENTS}

We thank the referee for useful suggestions. The second author has been supported by Grant No. MM-1003/2000 of the NFSR of Bulgaria.

\section{REFERENCES}

[1] N. A'Campo, Le groupe de monodromie du déploiement des singularités isolées de courbes planes. I. Math. Ann. 213 (1975), 1-32. MR 51:13282

[2] E. Arbarello, M. Cornalba, P.A. Griffiths, J. Harris, Geometry of Algebraic Curves, vol. 1, Springer, New York, 1985. MR 86h:14019

[3] V.I. Arnold, S.M. Gusein-Zade, A.N. Varchenko, Singularities of Differentiable Maps, vols. 1 and 2, Monographs in Mathematics, Birkhäuser, Boston, 1985 and 1988. MR 86f:58018, MR 89g:58024

[4] V.I. Arnold, Yu.S. Il'yashenko, Ordinary Differential Equations, in: Dynamical Systems I, Encyclopaedia of Math. Sci., vol. 1, Springer, Berlin, 1988.

[5] V.I. Arnold, Geometrical Methods in the Theory of Ordinary Differential Equations, Springer, New York, 1988. MR 89h:58049

[6] V.I. Arnold, Sur quelques problèmes de la théorie des systèmes dynamiques, Topological Methods in Nonlinear Analysis 4 (1994), 209-225. MR 96i:58001

[7] V.I. Arnold, Some unsolved problems in the theory of differential equations and mathematical physics, Russian Math. Surveys 44 (1989), no. 4, 157-171. MR 90m:00003

[8] V.I. Arnold, Ten problems, in: Theory of singularities and its applications, Adv. Soviet Math. 1, pp. 1-8, Amer. Math. Soc., Providence, 1990. MR 91k:58001

[9] E. Brieskorn, Die Monodromie der isolierten Singularitäten von Hyperflächen, Manuscripta Math. 2 (1970), 103-161. MR 42:2509

[10] A. Dimca, Singularities and Topology of Hypersurfaces, Springer, Berlin, 1992. MR 94b:32058

[11] A. Gasull, W. Li, J. Llibre, Zh. Zhang, Chebyshev property of complete elliptic integrals and its application to Abelian integrals, Pacific J. Math. 202 (2002), no. 2, 341-361. MR 2002m:34046

[12] L. Gavrilov, Isochronicity of plane polynomial Hamiltonian systems, Nonlinearity 10 (1997), no. 2, 433-448. MR 98b:58143

[13] L. Gavrilov, Petrov modules and zeros of Abelian integrals, Bull. Sci. Math. 122 (1998), no. 8, 571-584. MR 99m:32043

[14] L. Gavrilov, Abelian integrals related to Morse polynomials and perturbations of plane Hamiltonian vector fields, Ann. Inst. Fourier 49 (1999), no. 2, 611-652. MR 2000c:34081 
[15] L. Gavrilov, Nonoscillation of elliptic integrals related to cubic polynomials with symmetry of order three, Bull. London Math. Soc. 30 (1998), no. 2, 267-273. MR 99a:34077

[16] L. Gavrilov, The infinitesimal 16th Hilbert problem in the quadratic case, Invent. Math. 143 (2001), 449-497. MR 2002g:34069

[17] A.B. Givental, Sturm's theorem for hyperelliptic integrals, Leningrad Math. J. 1 (1990), 1157-1163. MR 91c:58038

[18] P.A. Griffiths, J. Harris, Principles of algebraic geometry, Pure and Appl. Math., John Wiley and Sons, New York, 1978. MR 80b:14001

[19] D. Hilbert, Mathematische probleme, Gesammelte Abhandlungen III, Springer-Verlag, Berlin (1935), pp. 403-479. MR 41:8201c

[20] E. Horozov, I.D. Iliev, Linear estimate for the number of zeros of Abelian integrals with cubic Hamiltonians, Nonlinearity 11 (1998), no. 6, 1521-1537. MR 99j:34036

[21] D. Novikov and S. Yakovenko, Tangential Hilbert problem for perturbations of hyperelliptic Hamiltonian systems, Electron. Res. Announc. Amer. Math. Soc. 5 (1999), 55-65 (electronic). MR 2000a:34065

[22] G.S. Petrov, Number of zeros of complete elliptic integrals, Funct. Anal. Appl. 18 (1984), 72-73; 20 (1986), 37-40; 21 (1987), 87-88; 22 (1988), 37-40; 23 (1989), 88-89; 24 (1990), 45-50. MR 89i:33002. MR 92c:33036

[23] L.S. Pontryagin, On dynamic systems close to Hamiltonian systems, Zh. Eksp. Teor. Fiz. 4 (1934), 234-238 (Russian).

[24] R. Roussarie, Bifurcation of Planar Vector Fields and Hilbert's sixteenth Problem, Progress in Mathematics, vol. 164, Birkhäuser, Basel, 1998. MR 99k:58129

Laboratoire Emile Picard, CNRS UMR 5580, Université Paul Sabatier, 118, route de Narbonne, 31062 Toulouse Cedex, France

E-mail address: 1.gavrilov@picard.ups-tlse.fr

Institute of Mathematics, Bulgarian Academy of Sciences, P.O. Box 373, 1090 Sofia, BULGARIA

E-mail address: iliya@math.bas.bg 